\title{
Landscape of IDH1/2 mutations in Chinese patients with solid tumors: a pan-cancer analysis
}

\author{
Dong Shen ${ }^{1}$, Junling Zhang ${ }^{2}$, Kai Yuan ${ }^{3}$, Jing Zhao ${ }^{2}$, Zhengyi Zhao ${ }^{2}$, Longgang Cui ${ }^{2}$, Yuzi \\ Zhang ${ }^{2}$, Guoqiang Wang ${ }^{2}$, Shangli Cai ${ }^{2}$, Yuezong Bai ${ }^{2}$, xiang huang ${ }^{4}$, and Wei $\mathrm{Li}^{4}$ \\ ${ }^{1}$ The Jiangyin Clinical College of Xuzhou Medical University \\ ${ }^{2} 3 \mathrm{D}$ Medicines Inc. \\ ${ }^{3}$ The Affiliated Changzhou No. 2 People's Hospital of Nanjing Medical University \\ ${ }^{4}$ Jiangsu Province People's Hospital and Nanjing Medical University First Affiliated \\ Hospital
}

July 6,2020

\begin{abstract}
Isocitrate dehydrogenase (IDH) is an enzyme family involved in cell aerobic metabolism of tricarboxylic acid cycle. However, the landscape of IDH mutations in pan-cancer has not been fully characterized. Tissue or blood samples were subjected to next-generation sequencing (NGS) for detection the IDH mutation. A total of 28.868 patients from more than 20 solid tumor species were analyzed. 374 cases (1.30\%) with IDH mutations were identified. Among all the IDH mutations cases, 80 (21.4\%) were biliary tract cancer, $80(21.4 \%)$ were lung cancer, $57(15.2 \%)$ were liver cancer and $42(11.2 \%)$ were colorectal cancer. The most common IDH variant were IDH1 and IDH2 which were discovered in $0.81 \%$ cases and $0.47 \%$ cases, respectively. TP53, PBRM1 and BAP1 were the most significantly mutated genes in biliary tract cancer which were different from others cancer. Moreover TMB were significantly higher in lung cancer, colorectal cancer and gastric cancer than biliary tract cancer ( $\mathrm{p}=$ 0.0164, $\mathrm{p}<0.0001, \mathrm{p}=0.0067$, respectively) and biliary tract cancer patients with IDH mutation had lower TMB compared with wild-type IDH. Somatic IDH mutation was found in multiple solid tumors and IDH would be a driver gene in biliary tract cancer.
\end{abstract}

\section{Introduction}

Isocitrate dehydrogenase (IDH) is an enzyme family involved in cell aerobic metabolism of tricarboxylic acid cycle, where $I D H 1$ and $I D H 2$ are commonly mutated metabolic genes in human cancers such as glioma and hematologic malignancies [1,2]. Since a missense mutation of $I D H 1$ was first identified in progressive glioblastoma in 2008 [3], numerous studies have been conducted to describe this genetic mutation and to understand its biological impact on tumors [4]. And it has been confirmed IDH1/2 mutations have been associated with prognostic and predictive values as biomarkers [5].

The understanding of the role of IDH1/2 mutations in tumorigenesis and early occurrence has led to the development of $I D H 1 / 2$ mutation inhibitors. IDH inhibitors have shown promising efficacy not only in hematologic malignancies, but also in cholangiocarcinoma patients harboring IDH1 mutations, such as AG-120 (NCT02073994), AG-881 (NCT02481154) and BAY1436032 (NCT02746081) [6-8]. However, the landscape of $I D H 1 / 2$ mutations in pan-cancer has not been fully characterized. In this study, we invested the IDH1/2 pathologic or likely pathologic mutations landscape in pan-cancer.

Material and Methods

Clinical Specimens 
Formalin-Fixed Paraffin-Embedded (FFPE) tumor sample or biopsy $(\mathrm{n}=28,868)$ of pan-cancer patients were enrolled in this study, including biliary carcinoma (1377 cases), liver cancer (2148 cases), lung cancer (11614 cases) and colorectal cancer (4056 cases), etc. The following clinical characteristics were abstracted: age, sex, and tumor histology.

\section{NGS Sequencing}

Tumor tissue samples (self-blood negative control) of pan-cancer were subjected to NGS for detection the IDH1/2 mutation with $800 \times$ sequencing depth in a College of American Pathologists (CAP) and Clinical Laboratory Improvement Amendments (CLIA) certified laboratory of 3D Medicines Inc. All pathologic or likely pathologic mutations of IDH1/IDH2 were considered. In addition, the TMB was defined as the total number of nonsynonymous mutation in coding region. This study was conducted under the approval of the ethics committees of the hospitals and informed consents were obtained from patients.

\section{Results \\ Patient Characteristics}

From January 01, 2017 to October 30, 2019, a total of 28,868 cases of Chinese solid tumor types were included in this study, including biliary carcinoma (4.77\%), liver cancer $(7.44 \%)$, lung cancer $(40.23 \%)$ and colorectal cancer (14.05\%). 374 cases $(1.30 \%)$ with $I D H$ mutations were identified. Patients with $I D H$ mutation $<60$ years old were $49.7 \%(\mathrm{n}=186)$. Among all the $I D H$ mutations cases, $80(21.4 \%)$ were biliary tract cancer, 80 (21.4\%) were lung cancer, 57 (15.2\%) were liver cancer, and $42(11.2 \%)$ were colorectal cancer (Table 1 ).

\section{IDH Mutation of Pan-Cancer}

The prevalence of IDH1/IDH2 mutations in 28.868 patients with different cancer types is summarized in Figure 1, with biliary tract cancer patients having the highest levels of IDH1/IDH2mutations (80/1377). Across all 28,868 patients, the mutational frequencies of $I D H 1$ and $I D H 2$ were $0.81 \%$ and $0.47 \%$, respectively.

We further investigated the genetic variation profile of $I D H$ mutated patients (Figure 2). Targeted therapies have been successfully developed to treat lung cancer harboring driver gene mutations. In lung cancer, driver gene mutations EGFR and KRAS were the most significantly mutated genes, whereas TP53, PBRM1 and $B A P 1$ were more frequently observed in biliary tract cancer (Fig. 2A, C ). In addition, TP53, PBRM1 and $B A P 1$ were high frequency mutated gene in liver cancer (Fig. 2B ). APC, TP53 and KRAS were among the top mutated genes in colorectal cancer, while TP53 .SPTA1 and ACVR2A were more in IDH mutated gastric cancer (Fig. 2D,E).

\section{Associate with TMB and IDH mutation}

We also analyzed the association between IDH1/IDH2 mutations and TMB in five type of cancer. As shown in Figure 2F . The TMB were significantly higher in lung cancer, colorectal cancer and gastric cancer than biliary tract cancer $(p=0.0164, p<0.0001, p=0.0067$, respectively). In addition, we also analyzed the relationship between IDH mutation/wild-type with TMB in biliary tract cancer in Figure 2G . Patients with $I D H$ mutation had lower TMB compared with patients with wild-type $I D H(p=0.0236)$.

\section{Discussion}

From a cohort of 28.868 patients with different types of solid tumors, a high frequency of IDH1/IDH2 mutations were observed not only in biliary tract cancer, but also liver cancer, lung cancer, colorectal cancer and others. The reprogramming of cellular metabolism is a fundamental characteristic of cancer, and IDH1/2 mutations represent key therapeutic targets in this arena. Somatic IDH1/2mutations are found in multiple solid tumors, and mounting evidence indicates that they contribute to premalignant disorders as well as early and late cancers. And then, another interesting point, we found that TMB was higher in other gastrointestinal tumors. However, in biliary tract cancer, IDH mutation accompanied by low TMB indicates that IDH would be a driver gene in biliary tract cancer. With the continuous emergence of IDH inhibitors, a considerable 
number of patients with solid tumors carrying IDH1/2 gene mutations may be more likely to benefit from IDH inhibitors, which is worth further expectation and exploration in clinical studies.

\section{Acknowledgments}

None.

Author Contributions Conception and design: Shen D, Zhang JL, Li W, Huang X. Analysis and interpretation of data: Zhang JL. Drafting the article: All authors. Huang X accepts full responsibility for the work and/or the conduct of the study, had access to the data, and oversaw the decision to publish.

\section{Funding}

None.

\section{Data Availability Statement}

The data that support the findings of this study are available from the corresponding author upon reasonable request.

\section{Ethics approval and consent to participate}

Tumor tissue samples (self-blood negative control) of pan-cancer were subjected to NGS n a College of American Pathologists (CAP) and Clinical Laboratory Improvement Amendments (CLIA) certified laboratory of 3D Medicines Inc. This study was conducted under the approval of the ethics committees of the hospitals and informed consents were obtained from patients.

\section{Consent for publication}

Not applicable.

Conflict of Interest Drs Junling Zhang, Jing Zhao, Zhengyi Zhao, Longgang Cui, Yuzi Zhang, Guoqiang Wang, Shangli Cai, Yuezong Bai are employees of 3D Medicines Inc. Other authors declare no conflict of interest.

\section{Reference}

1. Norsworthy KJ, Luo L, Hsu V, Gudi R, Dorff SE, Przepiorka D, Deisseroth A, Shen YL, Sheth CM, Charlab R, Williams GM, Goldberg KB, Farrell AT, Pazdur R (2019) FDA Approval Summary: Ivosidenib for Relapsed or Refractory Acute Myeloid Leukemia with an Isocitrate Dehydrogenase-1 Mutation. Clin Cancer Res . 25(11):3205-3209. doi:10.1158/1078-0432.CCR-18-3749

2. Yang H, Ye D, Guan KL, Xiong Y (2012) IDH1 and IDH2 mutations in tumorigenesis: mechanistic insights and clinical perspectives. Clin Cancer Res . 18(20): 5562-5571. doi:10.1158/1078-0432.CCR-12-1773

3. Parsons DW, Jones S, Zhang X, et al (2008) An integrated genomic analysis of human glioblastoma multiforme. Science . 321(5897):1807-1812. doi:10.1126/science.1164382

4. Turkalp Z, Karamchandani J, Das S (2014) IDH mutation in glioma: new insights and promises for the future. JAMA Neurol . 71(10):1319-1325. doi:10.1001/jamaneurol.2014.1205

5. Mondesir J, Willekens C, Touat M, de Botton S (2016) IDH1 and IDH2 mutations as novel therapeutic targets: current perspectives. J Blood Med . 7:171-180. doi:10.2147/JBM.S70716

6. Mellinghoff IK, Touat M, Maher E, DeLaFuente M, Cloughesy TF, Holdhoff M, Cote G, et al.(2016) ACTR-46. AG120, a first-in-class mutant IDH1 inhibitor in patients with recurrent or progressive IDH1 mutant glioma: Results from the phase 1 glioma expansion cohorts. Neuro Oncol . 18:vi12.

7. Mellinghoff I, Penas-Prado M, Peters K, Cloughesy T, Burris H, Maher E, Janku F, et al (2018) ACTR-31. Phase 1 study of AG-881, an inhibitor of mutant IDH1 and IDH2: Results from the recurrent/progressive glioma population. Neuro Oncol. 20:vi18. 
8. Pusch S, Krausert S, Fischer V, Balss J, Ott M, Schrimpf D, Capper D, et al (2017) Pan-mutant IDH1 inhibitor BAY 1436032 for effective treatment of IDH1 mutant astrocytoma in vivo. Acta Neuropathol.133:629644. doi:10.1007/s00401-017-1677-y

\section{Figure legends}

Figure 1 Prevalence of IDH1/IDH2 mutations in 28,868 patients with pan-cancer.

Figure 2 Top 20 significantly mutated genes in IDH1/2mutated patients of biliary tract cancer (A), liver cancer (B), lung cancer (C), colorectal cancer (D) and gastric cancer (E). Genes were ranked by mutation frequencies (right panel). Age and gender are annotated in the top panel. Tumor mutational load among IDH1/2mutated pan-cancers (F) and in biliary tract cancer $(\mathrm{G})$.

\section{Hosted file}

Table.docx available at https://authorea.com/users/337652/articles/463277-landscape-of-idh12-mutations-in-chinese-patients-with-solid-tumors-a-pan-cancer-analysis

\section{Type of cancer}

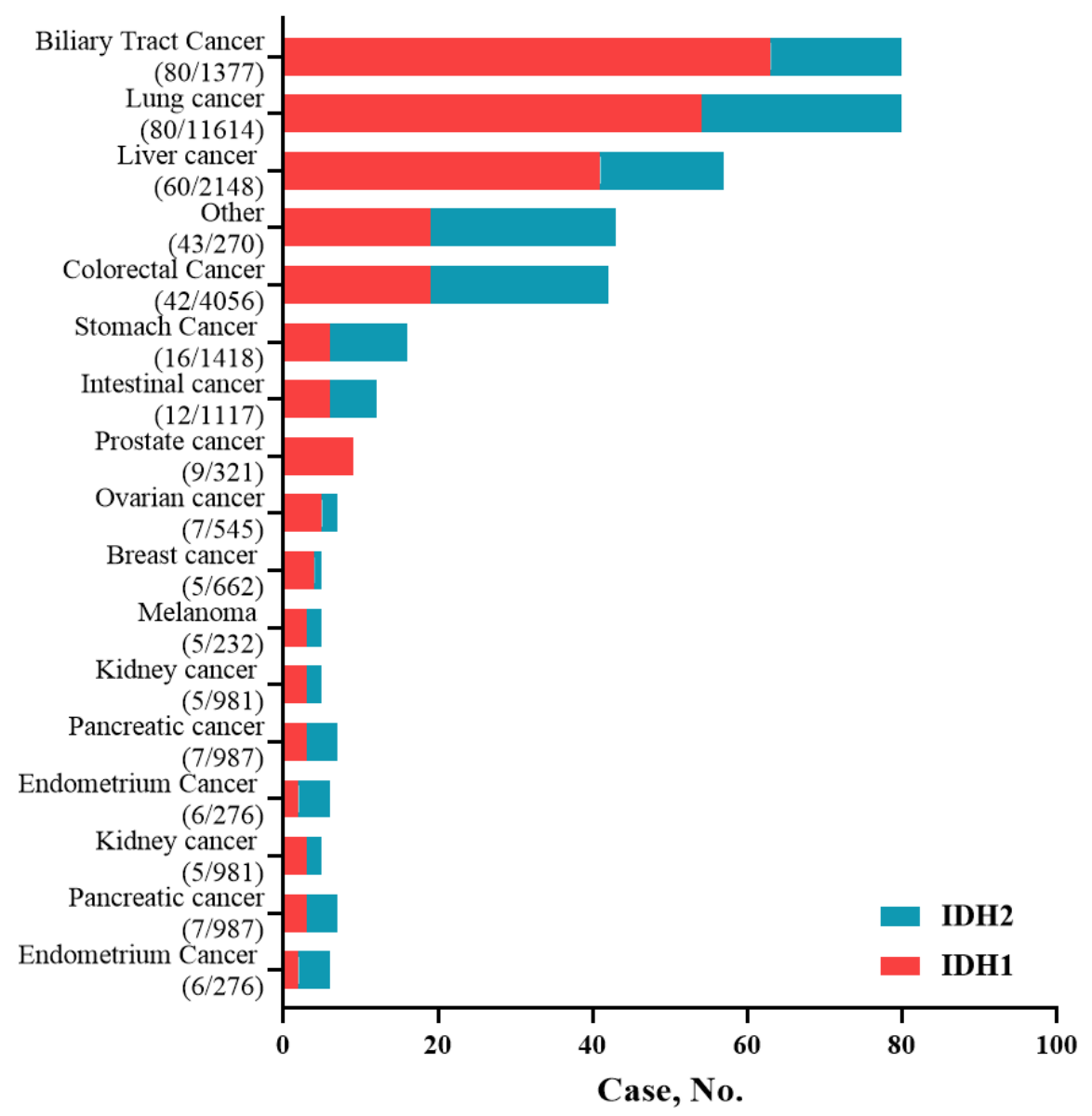




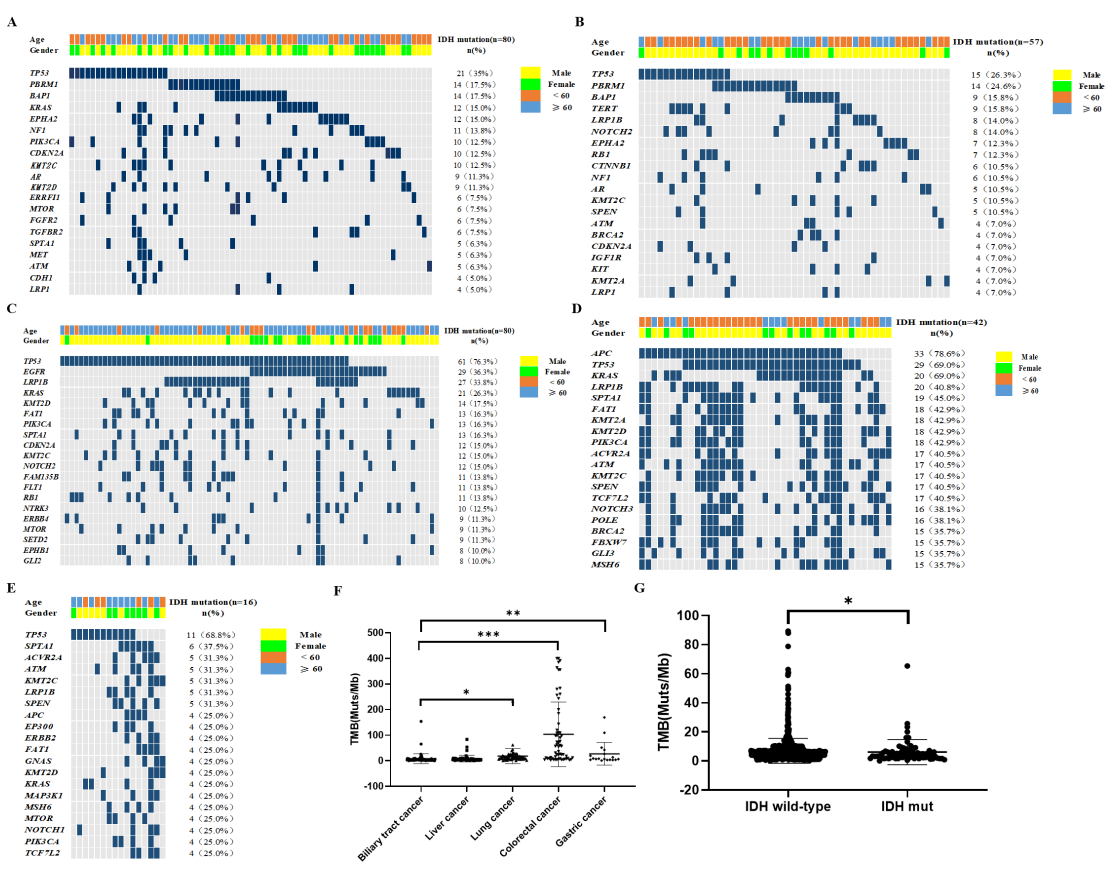

